\title{
Research on Train Over-taking Organization of Urban Rail Transit
}

\author{
Junjun Liu ${ }^{1}$, Baohua Mao² \\ ${ }^{1}$ MOE Key Laboratory for Urban Transportation Complex Systems Theory and Technology, Beijing \\ Jiaotong University, Beijing 100044, China \\ 2 Integrated Transportation Research Centre of China, Beijing Jiaotong University, Beijing 100044, \\ China
}

Keywords: increasing of express, departure interval, displays periodic variation.

\begin{abstract}
Study on train over-taking organization of urban rail transit and propose calculation methods of local train's waiting time as well as the length of the cross section. Combining with actual operation of urban rail transit, the effects of departure interval, tracking interval and the maximum operating speed difference of express/local train on local train's waiting time and the length of the cross section are analyzed. The result shows that with the increasing of express/local train's departure interval the local train's waiting time displays periodic variation. Under specific parameters, the cycle is 54s.In a single cycle, the waiting time increases linearly with the departure interval. If the tracking interval is within 90-150s and the departure interval is greater than 133s, the local train's waiting time increases with the increase of tracking interval. If the overtaking occurs in the section, the length of the cross section increases with the increase of the tracking interval. If the tracking interval increases from 90 s to 120 s, the length of cross section will increase by $25.00 \%$ when the maximum operating speed difference of express/local train is $0 \mathrm{~km} / \mathrm{h}$. And under the same tracking interval, the length of the cross section decreases with the increase of the maximum operating speed difference. If the maximum operating speed difference between express/local train increases from $0 \mathrm{~km} / \mathrm{h}$ to $20 \mathrm{~km} / \mathrm{h}$, the length of the cross section will decrease by $25.00 \%$ when the tracking intervals is $90 \mathrm{~s}$.
\end{abstract}

\section{Introduction}

Express/local operation is suitable for long distance suburban lines with large distances. It has practical significance for Chinese urban rail transit system, which is expanding continuously. Scholars in the field of transportation have done a lot of research on over-taking organization of Urban Rail Transit. Qingrui Zhou proposed the express train passed the local slow train at certain stations or intervals under the common line operation mode of the express/local train [1]. Saeed Zolfaghari, etc. confirmed that the average waiting time for passengers in the crossing station is prolonged[2].Li Zheng put forward an optimization model of single objective cross-stop train operation scheme with minimum total travel time[3]. Xinmiao Zhao studied the path selection of passenger perception time difference under the express train [4]. Baoguo Zhang studied the problem of the quantity and position about the overtaking station as well as impact on capacity [5]. Daoping Miu studied the influence of the auxiliary line of the common crossing station on the construction difficulty and the cost of the station [6].

The existing research mainly focus on the skip stop train plan optimization, passenger travel choice, the setting, number of stations, construction cost etc. Only a few documents put forward the problem of passenger waiting time extension at the crossing station, and there are few studies on the time of waiting and the length of the crossing interval. Based on the above research results, the over-taking organization process is studied. And the influence of the tracking interval , the departure interval of the express/local train and the maximum running speed of the express/local train have on local train's waiting and the length of cross section have been analyzed . 


\section{Train Over-Taking Organization}

According to the crossing location, the urban rail transit train over-taking can be divided into station and section. Stop time difference and interval time and other factors related. The location of the overtaking station or interval is related to the interval between the start of the express/local train, the difference between the interval operation, the time of the stop and the interval time. According to the operation characteristics of the express/local train, puts forward the following hypothesis: the express/local train is unbalanced, and the departure interval between the ahead slow train and the rear express train is $T_{l-e}$. In order to reduce the influence of the crossing station, the express train just skips one station. The express will skip-stop as it overtakes the slow train. Every local train is up to overtake once more, every express train overtakes local train once more most.

If the time interval ${ }^{a_{m}}$ the ahead slow train and the rear express train arrives at $\mathrm{M}$ station is greater than or equal to the tracking interval $I$ but the departure interval $h_{m}$ is less than $I$,M station should be set as overtaking station or the first stop of cross interval. If the departure interval $h_{m}$ is greater than or equal to $I$ but the time interval $a_{m+1}$ the ahead slow train and the rear express train arrives at $\mathrm{M}$ station is less than $I$,M station should be set as overtaking station or the first stop of cross interval.

\subsection{Local Train's Waiting Time}

If express train over-takes local train at $\mathrm{M}$ station, local train stops at the side track to avoid express train. After it passing through $\mathrm{M}$ station, the local train leaves the station. The waiting time of local train is shown in figure 1.

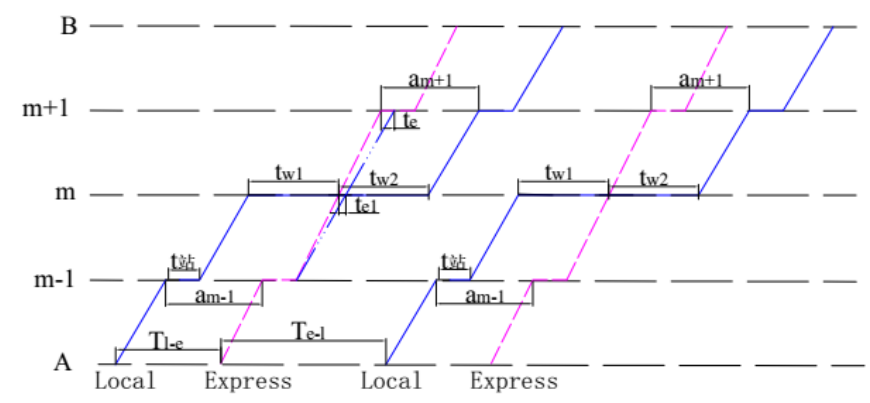

Figure 1. Schematic diagram of waiting time of local train

Local train collision waiting time calculation formula is (1) - (4).The formula (3) constrains the departure interval between the ahead slow train and the rear express train and the formula (4) restricts the time $t_{w 2}$ component of the slow train.

$$
\begin{array}{cc} 
& t_{\mathrm{w}}=t_{\mathrm{w} 1}+t_{\mathrm{w} 2} \\
& t_{\mathrm{w} 1}=T_{l-e}-(N-1)\left(t_{e}+t_{s}\right)-t_{e 1} \\
\text { s.t. } & T_{l-e}-(N-1)\left(t_{e}+t_{s}\right)-t_{e 1} \geq I \\
& \text { s.t. } \quad t_{\mathrm{w} 2} \geq I
\end{array}
$$

In the above formula $t_{w}$ refers to the local train's waiting time when the local train stops to avoid the express train and it consists of two parts: ${ }^{t_{w 1}}$ and ${ }^{t_{w 2}} . N$ refers to number of express train's crossing stations when it overtakes the local train. ${ }^{t_{e}}$ refers to the total running time to save when express train skip-stops one station and ${ }^{t_{e 1}}$ refers to the running time saved by the express train from the previous station to the crossing station. According to the operation results of the simulation experiment system of urban rail transit train [7], the ${ }^{t_{e}}$ value is about $24 \mathrm{~s}$ and ${ }^{t_{e 1}}$ values about $13 \mathrm{~s} .{ }^{t_{s}}$ refers to the stop time of express/local train, all of which are taken for $30 \mathrm{~s} . I$ refers to track train interval time, this paper assumes that the minimum tracking interval time is $90 \mathrm{~s}$.

\subsection{Length of Crossing Interval}

The $m$ station of a certain urban rail transit line is the first station in the crossing section, and there are a total of $k$ stations in the interval. From the station $m$ to $m+k-1$, the local train stops at the 
side track and the express train passes through the main line .The express and local train runs relatively independently and the tracking operation should be kept before the $m$ station and after the $m+k-1$ station, namely, $a_{m} \geq I, h_{m+k-1} \geq I$.

The express train completes the local train in $m$ to $m+k-1$ interval and the local train does not need to extend the stop time to avoid the express train. The formula for calculating the interval length is (5) - (7), the formula (6) and (7) constrain the express/local train track the operation outside the line.

$$
\begin{array}{ll} 
& L=\min \sum_{i=m}^{m+k-2} L(i, i+1) \\
& \text { s.t. } \quad a_{m} \geq I \\
\text { s.t. } \quad & \sum_{i=m}^{m+k-2} \Delta\left(t_{, i+1}\right)+k \cdot t_{s}-a_{m} \geq I
\end{array}
$$

In the above equation, $L$ refers to the length of the crossing interval, that is, the distance between the center lines of the first station and the end of the line. $L(i-1, i)$ refers to the distance between center line of the station $i$ and the station $i+1$ in the crossing interval. $\Delta t_{(i, i+1)}$ refers to the time difference between station $i$ and station $i+1$.

\section{Analysis on Waiting Time of Local Train}

It is assumed that the distance between urban rail transit stations is $1.5 \mathrm{~km}$ and above and express/local train's formation, speed grade is the same, the maximum running speed is $100 \mathrm{~km} / \mathrm{h}$ when express train overtakes local train at station. This chapter analyzes the local train's waiting time under different organization modes.

In order to ensure the track operation of the express/local train, the minimum interval between the ahead local train and the rear express train in the starting station is restricted by $N$.Under different tracking intervals, the relationship between the $N$ and $T_{l-e}$ is shown in Table 1.

Table 1. Relationship between $N$ and $T_{l-e}$ under different tracking intervals

\begin{tabular}{c|cccc}
\hline I & & & 3 & 4 \\
$T_{l-e}$ & 1 & 2 & & \\
$\mathrm{~N}$ & & & {$[211,265)$} & {$[265,319)$} \\
10 & {$[103,157)$} & {$[157,211)$} & {$[195,349)$} \\
150 & {$[133,187)$} & {$[187,241)$} & {$[241,295)$} & {$[325,379)$} \\
\hline
\end{tabular}

Table I shows when $I=90 \mathrm{~s}$ and $103 \leq T_{1-e}<157$, it is possible to determines that $N=1$.Combined type (1-2), local train's waiting time $t_{w}=T_{l-e}-13+t_{w 2}$.In order to reduce the influence of overtaking on the local train, this article takes $t_{w 2}=I$. Therefore, in this range, the local train's waiting time increases linearly with $T_{l-e}$. The minimum is $2 I$ and the maximum is close to $2 I+t_{e}+t_{s}$.If $157 \leq T_{l-e}<211$, in order to reduce waiting time, the express train will overtake the local train in the second skipping, and the waiting time will increase with the increase of $T_{l-e}$. The range of change will be $\left[2 I, 2 I+t_{e}+t_{s}\right)$. In summary, under different tracking intervals, the change rule of $t_{w}$ with $T_{l-e}$ is shown in figure 2.

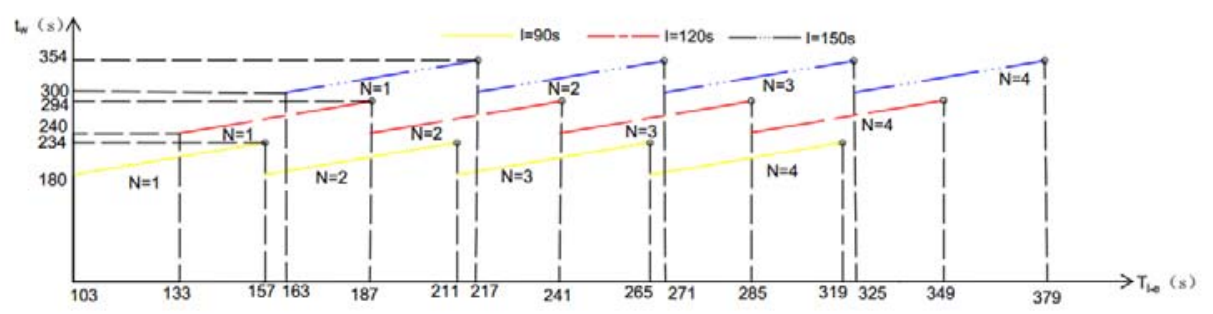

Figure 2. The changing rule of $t_{w}$ with $T_{l-e}$ under different tracking intervals 
The figure 2 shows that under the same track interval, the local train's waiting time $t_{w}$ changes periodically with $T_{l-e}$ and the change period is $T=t_{e}+t_{s}$.In the single period, $t_{w}$ increases linearly with $T_{l-e}$, and its variation range is $\left[2 I, 2 I+t_{e}+t_{s}\right)$.If $T_{l-e}$ is greater than the 133s, under the same departure interval, local train' waiting time $t_{w}$ increases with the tracking interval $I$.Net added value depends on the added value of the tracking interval, regardless of the size of the initial interval.

\section{Analysis on Length of Crossing Interval}

This chapter analyzes the influence of the maximum speed difference and the tracking interval on the length of the crossing interval. In order to reduce the influence of the crossing interval on the project cost, it designs $a_{m}=h_{\mathrm{m}+\mathrm{k}-1}=I$.In order to give full play to the advantages of long distance line spacing, the speed limit of the line is generally higher than $100 \mathrm{~km} / \mathrm{h}$, assuming that the local train's maximum operating speed is $100 \mathrm{~km} / \mathrm{h}$, the express train's is $100 \mathrm{~km} / \mathrm{h}$ or $120 \mathrm{~km} / \mathrm{h}$, and the tracking interval is 90,120 and 150 s.

Calculated by the urban rail transit train running results of simulation experiment system the pure running time difference between the express/local trains in one station is 22s. According to formula (5)-(7), the length of crossing interval under different tracking intervals is calculated. The results show that the length of crossing interval is $6.0,7.5,9.0 \mathrm{~km}$ when the tracking interval is 90,120 , 150 s. With the increase of the tracking interval, interval length is increased by $25.00 \%$ and $20.00 \%$.

While the maximum operating speed of the express train is $120 \mathrm{~km} / \mathrm{h}$ but the local train's is $100 \mathrm{~km} / \mathrm{h}$ the pure running time difference between the express/local train in one station varies with the distance between stations as shown in table 2 .

Table 2. Pure running time difference between the express/local trains

$\begin{array}{cccccccccc}L_{(i, i+1)} & (\mathrm{km}) & 1.5 & 2.0 & 2.5 & 3.0 & 3.5 & 4.0 & 4.5 & 5.0 \\ \Delta t_{(i, i+1)} & (\mathrm{s}) & 33 & 37 & 41 & 45 & 48 & 53 & 55 & 60\end{array}$

Combined with table 3-1 and formula (5) - (7), the length of crossing interval under different tracking intervals is calculated. The results show that the length of crossing interval is $4.5,6.0,7.5 \mathrm{~km}$ when the tracking interval is $90,120,150$ s. As the tracking interval increased, the length of the crossing interval increases by $33.33 \%$ and $25.00 \%$ in turn.

While the maximum operating speed difference of express/local train is increased from 0 to $20 \mathrm{~km} / \mathrm{h}$, and the length of the crossing interval decreases by $25.00 \%, 20.00 \%$ and $16.67 \%$, respectively, when the tracking interval is 90,120 and 150s. Therefore, when the tracking interval is within 90-150s, the length of the crossing interval is reduced with the increase of the maximum operating speed of the express/local train, and the larger the tracking interval is, the smaller the range of the length of the crossing interval.

\section{Conclusion}

1) Put forward calculation methods of local train's waiting time and the length of the crossing interval.

2) While express trains overtake local train at station, the local train' waiting time $t_{w}$ changes periodically with $T_{l-e}$ and the change period is $54 \mathrm{~s}$ under the parameters set in this paper. In the single period, $t_{w}$ increases linearly with $T_{l-e}$, and its variation range is $\left[2 I, 2 I+t_{e}+t_{s}\right)$. Tracking interval in the range of 90-150s, if $T_{l-e} \geq 133 \mathrm{~s}$, under the same departure interval, $t_{w}$ increases with the tracking interval increase, net value added directly determined by the change of tracking interval.

3) While express trains overtake local train at crossing interval, the length of the interval increases with the growth of the tracking interval, and the greater the velocity difference, the greater the increase under the same maximum operating speed difference of express/local train. While the maximum speed difference of the express/local train is $0 \mathrm{~km} / \mathrm{h}$, the length of the crossing interval is increased by $25.00 \%$ and $20.00 \%$ respectively if he tracking interval is 90,120 and 150 s. While the 
speed difference is $20 \mathrm{~km} / \mathrm{h}$, the length of the crossing interval is increased by $33.33 \%$ and $25.00 \%$ respectively. Under the same tracking interval, the length of the crossing interval decreases with the increase of the maximum operating speed difference of the express/local train, and the larger the tracking interval is, the smaller the reduction is. Under the setting parameters of the article, if the maximum operating speed difference of the express/local train is increased from $0 \mathrm{~km} / \mathrm{h}$ to $20 \mathrm{~km} / \mathrm{h}$, the length of the crossing interval is reduced by $25.00 \%, 20.00 \%$ and $16.67 \%$, respectively, when the tracking interval is 90, 120 and 150s.

\section{Acknowledgements}

Corresponding author: bhmao@bjtu.edu.cn

This research work was financed by National Natural Science Foundation of China (No.71390332), The Fundamental Research Funds of Ministry of Education of China (No.2015JBZ010).

\section{References}

[1]. Qingrui Zhou.2013. “Analysis of the Express/Slow Trains Operation Mode for Urban Rail Transit of World Cities,” Urban Rapid Rail Transit, 26(2):18-22.

[2]. Saeed Zolfaghari, Nader Azizi, Mohamad Y. Jaber. 2004. “A model for holding strategy in public transit systems with real-time information,”J. International Journal of Transport Management, 2 (2):99 - 110.

[3]. Li Zheng and Sui Song and Shiwei He.2009. "Optimization Model and Algorithm of Skip-stop Strategy for Urban Rail Transit,”J.JOURNAL OF THE CHINA RAILWAY SOCIETY, 31(6):1-8.

[4]. Xinmao Zhao and Quanxin Sun and Ying Ding.2016. "Passenger Choice Behavior for Regional Rail Transit under Express/Local Operation with Overtaking, ”Journal of Transportation Systems Engineering and Information Technology, 16(5):104-109.

[5]. Guobao Zhang and Jia Fu and Mingshu Liu.2005. "Research on Skip - Stop Running Patterns of Urban Rail Transit and the Derived Problem of Train Overtaking, ”Urban Rapid Rail Transit, 18(5):18-22.

[6]. Daoping Miao.2015. "Research on the Scheme for the Station Sidings in the Express/Slow Metro Train Organization Mode, ”Journal of Railway Engineering Society, 32(06):97-101+105.

[7]. Jianfeng Liu and Ding Yong and Haidong Liu.2005. "Multi-train Movement Simulation System for Urban Rail Transit, ”Journal of Transportation Systems Engineering and Information Technology, 5(1):79-82. 